Revista Educación

ISSN: 0379-7082

ISSN: 2215-2644

revedu@gmail.com

Universidad de Costa Rica

Costa Rica

\title{
La recolección, utilización y almacenamiento de datos biométricos sensibles en deportistas: insumos para la carrera de Educación Física
}

Moncada-Jiménez, José; Salicetti-Fonseca, Alejandro; Carazo-Vargas, Pedro; Morera-Siércovich, Pier Luigi

La recolección, utilización y almacenamiento de datos biométricos sensibles en deportistas: insumos para la carrera de Educación Física

Revista Educación, vol. 45, núm. 1, 2021

Universidad de Costa Rica, Costa Rica

Disponible en: http://www.redalyc.org/articulo.oa?id=44064134030

DOI: https://doi.org/10.15517/revedu.v45i1.41607

\section{(c) $(1) \Theta(9$}

Esta obra está bajo una Licencia Creative Commons Atribución-NoComercial-SinDerivar 3.0 Internacional. 


\section{La recolección, utilización y almacenamiento de datos biométricos sensibles en deportistas: insumos para la carrera de Educación Física}

\section{Collection, Use and Storage of Sensitive Biometric Data for Athletes: Inputs for Physical Education Majors}

José Moncada-Jiménez

Escuela de Educación Fisica y Deportes, Universidad de

Costa Rica, Costa Rica

jose.moncada@ucr.ac.cr

iD https://orcid.org/0000-0001-9807-5163

Alejandro Salicetti-Fonseca

Costa Rica, Escuela de Educación Física y Deportes,

Universidad de Costa Rica., Costa Rica

alejandro.salicetti@ucr.ac.cr

iD https://orcid.org/0000-0001-7605-1128

Pedro Carazo-Vargas

Escuela de Educación Física y Deportes, Universidad de

Costa Rica, Costa Rica

pedro.carazo@ucr.ac.cr

(iD https://orcid.org/0000-0002-5374-7031

\section{Pier Luigi Morera-Siércovich}

Escuela de Educación Física y Deportes, Universidad de

Costa Rica, Costa Rica

psiercovich@yahoo.it

Recepción: 08 Mayo 2020

Aprobación: 02 Octubre 2020

\section{ReSUMEN:}

Recientes avances tecnológicos, incluyendo espacios de almacenaje y algoritmos computacionales, permiten la recolección y almacenaje de una amplia cantidad de variables biométricas asociadas al rendimiento deportivo. El propósito de este ensayo es reconocer la importancia de la tecnología portátil en el ámbito del deporte, así como las leyes internacionales y nacionales que regulan la recolección, uso y almacenaje de datos biométricos de naturaleza sensible provenientes de deportistas, con un ejemplo específico para el caso de futbolistas profesionales costarricenses. La información presentada y discutida es un insumo relevante y actual para la formación de profesionales en Educación Física. A diferencia de la normativa internacional, se concluye que en Costa Rica no existe claridad acerca de la legislación relacionada con la biometría del deporte. También queda en evidencia que es necesaria la creación de programas educativos interdisciplinarios en analítica deportiva.

Palabras Clave: Regulaciones, Leyes, Dispositivos portátiles, Wearables, Deportes.

\section{Abstract:}

Recent technological advances, such as storage capacity and computational algorithms, allow a large quantity of biometric variables associated with sports performance to be collected. This study addresses the relevance of wearable or portable technology in sports and its association to international and local laws that regulate the recollection, use and storage of sensitive biometric data from professional athletes, analyzed, specifically, within the context of Costa Rican professional soccer players. As opposed to international regulations, Costa Rica lacks specific laws regulating sports biometrics. Interdisciplinary educational programs for sports analytics are also required to provide insight into this topic, so relevant to Physical Education majors, today.

Keywords: Regulations, Laws, Portable Devices, Wearables, Sports. 


\section{INTRODUCCIÓN}

La computación y el internet han cambiado radicalmente la vida de las personas. Las computadoras ahora tienen el potencial para procesar cantidades inmensas de datos en fracciones de segundo, y el internet recolecta información cada segundo del día. La combinación de potentes computadoras y del internet ha hecho que existan personas programadoras capaces de crear algoritmos para la recolección y predicción de comportamientos de la población usuaria (Emerging Technology from the arXiv, 2016). Además, la inteligencia artificial ha permitido detectar y reconocer patrones provenientes de grandes cantidades de datos (Big data), ya sean estructurados (cédula de identidad, sexo, ingresos económicos) o no estructurados (la voz de una persona, el movimiento de una persona), para ponerlos al servicio de otros/as usuarios/as o compañías (Facebook, Google) y predecir futuros comportamientos, como por ejemplo, patrones de consumo de bienes y servicios o incluso del rendimiento deportivo (Isson, 2018).

El propósito de este ensayo es reconocer la importancia de la tecnología portátil en el ámbito de la educación física y el deporte, así como analizar las leyes que regulan la recolección, uso y almacenaje de datos biométricos de naturaleza sensible provenientes de deportistas, específicamente de futbolistas profesionales costarricenses. Adicionalmente, se desea demostrar la carencia de una oferta educativa universitaria en el área de analítica deportiva.

\section{ANALÍtiCA DEPORTIVA}

La tecnología de los dispositivos portátiles ha cambiado el mundo del deporte (Emerging Technology from the arXiv, 2016). A este tipo de dispositivos, se les define como "tecnologías electrónicas o computadoras que se incorporan a elementos de las prendas de vestir y accesorios, los cuales pueden llevarse cómodamente en el cuerpo" (Jhajharia, Pal y Verma, 2014, p. 5700). Estos dispositivos se encargan de medir y grabar, y algunos de éstos de transmitir, en tiempo real, una amplia cantidad de variables biométricas de la persona, que luego serán utilizadas con algún propósito particular. Incluso, estos dispositivos pueden desplegar y presentar, en tiempo real, una miríada de información, lo cual proporciona datos valiosos al atleta o su cuerpo técnico (Di Tore, 2015).

Debido al auge de estas tecnologías y a la gigantesca capacidad de procesamiento y almacenamiento de datos, diferentes instituciones de enseñanza superior crearon el campo de la analítica deportiva (Emerging Technology from the arXiv, 2016). Morgulev, Azar y Lidor (2018) la definen como “(...) la investigación y modelaje del rendimiento deportivo, que implementa técnicas científicas” (p.214), pero más específicamente como:

el manejo estructurado de datos históricos, la aplicación de modelos analíticos predictivos que utilizan esos datos, y la utilización de sistemas de información, con el propósito de informar a quienes toman decisiones y que permiten ayudarles a sus organizaciones a ganar una ventaja competitiva en el campo de juego (p. 214).

Esto significa que para los profesionales en análisis de datos se creó un nuevo nicho de empleo, en donde, por ejemplo, en EE. UU. el $23 \%$ de los equipos de la National Hockey League (NHL) contratan a este tipo de profesionales, lo cual contrasta con la National Football League (NFL) (56\%), la National Basketball Association (NBA) (80\%), y la Major League of Baseball (MLB) (97\%) (Isson, 2018). De acuerdo con la información publicada en el sitio web de la Major League of Soccer (MLS) (Major League of Soccer, 2019), aproximadamente $58 \%$ de los equipos poseen personal técnico especializado en el análisis de datos en los equipos profesionales de fútbol (data analyst, soccer analytics and data, sports medicine and science, sport scientist).

En Costa Rica, equipos como el Deportivo Saprissa, el Club Sport Herediano y la Liga Deportiva Alajuelense, así como la Federación Costarricense de Fútbol, poseen la tecnología para la recolección de 
datos biométricos, pero no el personal especializado en la captura, procesamiento, reducción y el análisis de datos. Aunque la formación de especialistas en el área no ha comenzado en Costa Rica, en otros países se ofrece educación universitaria como una especialidad dentro de un programa de Ciencias del Movimiento Humano y Administración Deportiva, así como programas completos de grado y posgrado (Discover Data Science, 2020). Estos programas universitarios combinan procesos de enseñanza-aprendizaje que incluyen la matemática, álgebra, computación e informática, el deporte, y la administración, entre otras. Esto significa que las universidades han detectado una necesidad y han implementado estrategias para crear ofertas educativas con alto potencial para la inserción en el mercado laboral.

\section{BiOMETRÍA DEPORTIVA}

La biometría en el deporte se refiere a la medición, recolección o grabación de variables físicas, por ejemplo: antropométricas (peso, estatura), fisiológicas (frecuencia cardiaca, fuerza, potencia, velocidad, sueño, variabilidad de la frecuencia cardiaca), biomecánicas (aceleración, saltos, caídas), psicológicas (ansiedad, vigor, fatiga), y conductuales (alimentación, actividad física), entre otras. Estas y otras variables forman parte integral de la disciplina de la Educación Física, y aunque el uso de la biometría deportiva es reciente y novedoso en algunos deportes, en otros como el baseball se han recolectado innumerables datos individuales durante más de 50 años (Hattery, 2017; Isson, 2018).

Existe una amplia variedad de dispositivos portátiles o wearables que permiten grabar información biométrica de los atletas (Polar., WIMU., Catapult., WHOOP., Zephyr., Motus., Fitbit.), en donde algunos de éstos tienen la capacidad de grabar en cada atleta la cantidad de 1000 datos por segundo de la variable o del grupo de variables de interés (Arnow, 2016; Osborne, 2017). Algunos dispositivos poseen dimensiones similares a las de un reloj de pulsera, lo que permite su cómoda utilización durante el entrenamiento, la competencia e incluso durante los periodos de recuperación; es decir, se pueden utilizar durante 24 horas. Es común observar a estudiantes y docentes de la carrera de Educación Física portar este tipo de dispositivos, ya que forman parte normal de los procesos de enseñanza y aprendizaje. Se espera que para el año 2020 el total de ventas para dispositivos portátiles relacionados con el acondicionamiento físico y el deporte, alcance un valor cercano a los $\$ 6000$ millones (Lamkin, 2016).

En el año 2019, un medio de comunicación reportó que el preparador físico de la Selección Nacional de Fútbol mayor de Perú utilizó una metodología de trabajo con tecnología inercial que incluía el uso de Global Positioning System (GPS) para controlar totalmente el estado físico de cada jugador de la selección (Rotnitzky, 2019). Además, se indica que el preparador físico usó la información recolectada para brindarle retroalimentación a cada jugador acerca de las probabilidades de su desempeño contra otros jugadores. El preparador físico afirmó que:

Con el trackeo descubrimos dos puntos determinantes: 1) Si superamos al rival por encima de un 4\% en los metros totales, sacamos el $65 \%$ de los puntos en juego. 2) Cuando superamos al contrario por más de un 12\% en los esfuerzos de más de 24 kilómetros por hora, en esfuerzos cercanos a la máxima intensidad, también sacamos el 65\% de los puntos en juego (Rotnitzky, 2019, párr. 8).

Aunque la entrevista no reveló el origen de los datos del equipo contrario para que el preparador físico pudiera realizar comparaciones con el rival, o si se analizaron múltiples variables; es evidente que actualmente se puede inferir que algunos de los cuerpos técnicos de los equipos de fútbol, así como de otros deportes, toman decisiones utilizando datos proporcionados por la tecnología. Este tipo de tecnología permite conocer la posición de cada atleta en el terreno de juego y puede calcular inclinación, caída libre, tiempo en el aire, aceleración, impacto, tiempo de reacción, saltos, golpes, velocidad angular, rodamientos, inclinación, y dirección de los movimientos (adelante, atrás, lateral), calorías gastadas y calidad de sueño, entre otros (Grow y Shackelford, 2020). 
Con estos instrumentos, se pueden registrar los valores de variables absolutas y relativas de cada atleta, por ejemplo; la distancia total recorrida, el porcentaje de la distancia recorrida a una intensidad definida por el usuario (alta velocidad $=35-36 \mathrm{~km} / \mathrm{h})$, distancias relativas al tiempo $(\mathrm{m} / \mathrm{min})$, y aceleraciones absolutas y relativas al tiempo, entre otras (Inglés, Bastida y Gómez, 2018). Es decir, el análisis científico de grandes cantidades de información les permite a las y los usuarios finales aplicar dicho conocimiento en su labor diaria de planeamiento y, posiblemente, estudiar y predecir comportamientos tácticos de sus atletas y de sus rivales (Rein y Memmert, 2016), así como explicar y predecir la aparición de lesiones (Kampakis, 2016; Rossi et al., 2018), predecir el éxito en una liga de baloncesto de alto nivel por medio de técnicas de análisis de inteligencia artificial, tales como el aprendizaje de máquinas - machine learning- o aprendizaje automatizado (Kannan, Kolovich, Lawrence y Rafiqi, 2018), o para reclutar jugadores y jugadoras jóvenes durante visorías (scouting) (Morgulev et al., 2018).

Por ejemplo, en el deporte del hockey sobre césped de nivel universitario, se utilizaron datos biométricos de los jugadores y se encontró, por medio de un modelo predictivo, que entrenar con una elevada carga cardiovascular dos días antes de la competencia predecía ganar el juego (Blanchfield et al., 2019). Los datos biométricos que utilizaron de cada jugador fueron la frecuencia cardiaca, distancia recorrida, velocidad, aceleración, cadencia e intervalos R-R. Por lo tanto, se podría argumentar que no solamente el cuerpo técnico de un equipo, sino también el propio deportista, se beneficiaría al conocer la información que proporcionan estos dispositivos, pues le permitiría saber si se encuentra cerca o al máximo de sus capacidades físicas (Brush, 2015). Para estudiantes de Ciencias del Movimiento Humano, poder contar con datos propios o de sus clientes o deportistas, les permitiría reforzar conocimientos teóricos adquiridos y ponerlos en práctica en escenarios de la vida real, favoreciendo su proceso de aprendizaje.

Como se mencionó, algunos de estos dispositivos contienen un sistema de posicionamiento global ( Global Positioning System, GPS), sistema de identificación de rastreo por radiofrecuencia (RFID), acelerómetros y giroscopios, y cada unidad se coloca dentro de un chaleco que cada atleta utiliza bajo la camisa de competencia oficial, de manera que no es visto por el público y no interfiere con el juego. Este es un lugar seguro para la integridad física del atleta y con una gran exposición a la señal satelital. Al finalizar el juego, el dispositivo se coloca en una estación que adquiere los datos y los procesa para luego ser exportados en formato commaseparated values (CSV) o de hoja de cálculo (Excel) y ser utilizados para el análisis estadístico. En algunos de estos dispositivos, la cantidad de variables que se obtienen por cada jugador en cada juego son superiores a las 120; y se pueden agrupar en aquellas relacionadas con distancia, aceleración, sprints o carreras cortas, impactos horizontales, pasos y saltos, y carga de trabajo del jugador.

En este momento, el estado del conocimiento en el deporte del fútbol se encuentra en una fase exploratoria para determinar si una, varias, o bien una combinación o asociación de variables, explican los datos (Blanchfield et al. 2019; Kampakis, 2016; Kannan et al., 2018; Rein y Memmert, 2016). Por ejemplo, no se sabe aún, consistentemente, si una sola variable como la distancia recorrida en la cancha es la que mejor explica la probabilidad de ganar, empatar o perder un juego. Tampoco se sabe, si la combinación de variables como la distancia recorrida junto con las aceleraciones representan un mejor modelo para explicar el rendimiento en un juego que otro grupo de variables como la velocidad y los saltos verticales. Lo que sí se sabe con certeza es que de cada atleta hay un expediente con millones de datos provenientes de entrenamientos y juegos, que en el futuro podrían significar la diferencia entre adquirir un contrato justo o incluso quedar sin empleo.

En este contexto, surge la interrogante de si en la legislación costarricense o internacional existe algún tipo de guía para saber a quién pertenecen los datos recopilados; es decir, si a jugadores y jugadoras de fútbol, al equipo u organización deportiva que realizó las contrataciones, o a ambos. O como lo cuestiona Greenwald (2017), ¿quién es dueño de los datos?

La Federación Internacional de Fútbol Asociación (FIFA), aprobó el uso de esta tecnología a partir del Campeonato Mundial de Fútbol del año 2014 (https://football-technology.fifa.com/es/media-tiles/ fifa-epts-wearables-licensees-and-certified-products/). La National Collegiate Athletic Association (NCAA), 
organización que regula el deporte universitario (no profesional) en los EE.UU., aprobó el uso de la tecnología portátil personal durante los juegos, con el único propósito de monitorear la salud y seguridad de las y los atletas (Arnold y Sade, 2017). De igual forma, la MLB aprobó el uso de este tipo de dispositivos (Lemire, 2016). Por lo tanto, existe una reciente tendencia a que las organizaciones deportivas cada vez comprendan la importancia y el posible valor financiero de la captura de información de cada atleta.

Sin embargo, actualmente existe un debate ético acerca de elementos como la validez, validez predictiva, cantidad, privacidad y seguridad de toda la información recopilada (Saxon, 2017; Sikka, Baer, Raja, Stuart y Tompkins, 2019; Socolow y Jolly, 2017), así como de aspectos relacionados con la adquisición de grandes volúmenes de información (big data) (Davalos, 2017; Greenwald, 2017; Hand, 2018; Jessop y Baker III, 2019; Karkazis y Fishman, 2017; Shahmiri, 2016), e incluso el marco conceptual en el que se deben manejar estas cantidades de información (Di Tore, 2015). Algunas empresas han cambiado de estrategia de mercado (Nike., Under Armour.), y ya no les interesa principalmente la creación y venta de dispositivos, sino la creación de algoritmos computacionales para reconocer patrones de las variables biométricas que puedan predecir el consumo de cada atleta y su rendimiento deportivo (Jessop y Baker III, 2019).

Adicionalmente, se discute acerca de posibles conflictos de interés de empresarios y representantes de organizaciones deportivas con compañías fabricantes, y algunas veces patrocinadoras de estas organizaciones, con respecto al registro y uso de la información biométrica recopilada de las y los deportistas (Karkazis y Fishman, 2017). También se discute acerca de juego limpio (Fairplay) respecto a quienes pueden tener acceso a la tecnología y quiénes no, pues obviamente pondría en desventaja a quienes tienen oportunidades limitadas de acceder a tecnología de vanguardia (Greenbaum, 2018). Pero aún más relevante es la recopilación de los datos bajo mecanismos coercitivos (Karkazis y Fishman, 2017), por ejemplo, cuando el entrenador del equipo exige los datos del jugador para tomar una decisión de si juega como titular o si va al banco de los suplentes, o en el peor de los casos, para no ser llamado para el juego o ser despedido. Este escenario también se podría extender a otros miembros del cuerpo técnico, como el personal médico, preparadores físicos, rehabilitadores e incluso los propios jugadores a través de la presión de pares (peer-pressure) (Arnold y Sade, 2017).

Desde el punto de vista de la investigación con datos provenientes de dispositivos de este tipo, apenas se están emitiendo recomendaciones o buenas prácticas para realizar investigación (Harriss, MacSween y Atkinson, 2019), aunque ya sus riesgos han sido discutidos anteriormente (Vermeulen y Venkata, 2018). En ese contexto, se recomienda que la persona atleta firme un formulario de consentimiento informado, en el que se le explique al menos para qué se van a utilizar los datos y cómo se va a garantizar su privacidad y la seguridad de esos datos. Además, se le debe explicar claramente que no firmar ese consentimiento tampoco traerá consecuencias negativas o que en cualquier momento puede solicitar que sus datos no se usen más. En esencia, y como lo menciona Hattery (2017), es el derecho a saber.Right to Know), o bien se podría decir que es un derecho de autonomía personal. Éste y otros temas éticos y morales, deben ser incorporados en los cursos de investigación universitarios, de manera que la discusión de la literatura pertinente y de casos concretos fortalezcan los procesos de aprendizaje.

\section{Datos de carÁcter Personal en Europa y Norteamérica}

De acuerdo con Gil (2016), un dato de carácter personal se define como "cualquier información concerniente a personas físicas identificadas o identificables" (p. 45). De la misma manera, indica que "Una persona es identificable cuando su identidad pueda determinarse, directa o indirectamente, mediante cualquier información referida a su identidad física, fisiológica, psíquica, económica, cultural o social, salvo que dicha identificación requiera actividades o plazos desproporcionados" (Gil, 2016, p. 45). Así, un argumento en contra de la inclusión de información biométrica podría ser el caso de variables genéticas, por medio de las cuales se podría saber la propensión a algún tipo de lesión o condición de salud de cada atleta. Es decir, realizar 
pruebas genéticas a atletas antes de su contratación podría considerarse negativo en tanto que discrimine a quienes poseen algún tipo de característica no deseada por el club.

En los EE. UU., de acuerdo al Genetic Information Nondiscrimination Act, es prohibido realizar pruebas genéticas para conseguir un empleo (Chapman, Mehta, Parent y Caplan, 2019), y eso es especialmente relevante para atletas profesionales, pero no aplica para estudiantes-atletas (Lazan y Greenbaum, 2017). En un sentido similar, la FIFA dispone que "La validez de un contrato no puede supeditarse a los resultados positivos de un examen médico..." (Fédération Internationale de Football Association, 2020, p. 23). Sin embargo, se han documentado casos en los que debido a que una prueba electrocardiográfica resulta irregular, se cambia la posición de escogencia de un jugador en la NFL (ranking de selección), o que, debido a pruebas médicas, algunos jugadores de la NBA no fueran seleccionados en la primera ronda de elección (draft) (Henne, 2017). Incluso en ausencia de un examen médico, un club podría tener una extensa cantidad de datos con los cuales podría predecir el rendimiento de un jugador; datos que precisamente no se sabe de quién son. Es decir, ¿son esos datos de carácter personal, de acuerdo con la definición de Gil González, son del patrono (club de fútbol), o se deberían negociar en los contratos (agentes de jugadores)? De acuerdo con la Corte Suprema de Justicia de Canadá, los datos biométricos pertenecen a la organización que los recolecta y no a las y los atletas (Casher, 2019); sin embargo, aclara que esos datos tuvieron que haber sido recolectados con el consentimiento informado del y la atleta.

En la Unión Europea, existe la General Data Protection Regulation, que indica que “...las personas naturales deberían tener el control de sus datos personales”, lo que se interpreta como un concepto de propiedad implícito (Schulz y Hennis, 2016, p. 2). Por otra parte, si el jugador decide que son datos protegidos, entonces hay legislación que le beneficia. Por ejemplo, en Europa se le considera como un derecho fundamental de acuerdo al artículo 16 del Tratado de Funcionamiento de la Unión Europea y el artículo 8 de la Carta de los Derechos Fundamentales de la Unión Europea (Gil, 2016). Un jugador o una jugadora de fútbol (o de cualquier deporte), sin embargo, podría dar un consentimiento específico para el uso de ciertos datos que considere que no violan su privacidad, y que, en términos generales, pueden ser beneficiosos para su salud y su desempeño deportivo, y que además le proporcionan más beneficios que riesgos (Roberts, Cohen, Deubert y Lynch, 2016). En todo caso, ese consentimiento debe ser informado, libre y sin coacción. De acuerdo con Henne (2017), todavía se necesita tener un conocimiento más profundo acerca de cómo los y las jugadoras perciben y valoran la información de su autonomía personal e integridad corporal, así como de su privacidad. Este tipo de estudios se podrían realizar por medio de colaboraciones entre universidades, federaciones de fútbol y asociaciones de deportistas profesionales para ir adoptando buenas prácticas que beneficien a las partes involucradas. De esa manera podrían alcanzar dos objetivos; por una parte, generar información autóctona, y por otra, reforzar el proceso de aprendizaje del alumnado a través de la investigación.

En otro escenario se podría argumentar que los datos pertenecen al patrono (club) únicamente durante el horario de trabajo (entrenamientos, juegos, viajes), pero no en el tiempo libre de la persona trabajadora (atletas). De esta forma, la empresa o club guardaría la información para futuras contrataciones, comparación con otros deportistas, $\mathrm{u}$ otras actividades que le permitan generar recursos financieros para la organización (promoción de atletas, mercadeo). Sin embargo, el tiempo de custodia y la seguridad del almacenamiento de esos datos continuarán siendo un tema por resolver, ya que se han descrito casos en los que ha habido violaciones a la seguridad (intrusiones, hackeo) a organizaciones como la World Anti-Doping Agency (WADA) y el equipo de baseball de los Astros de Houston en EE. UU. (Greenwald, 2017).

En eventos de carácter público, como la transmisión de juegos en torneos locales o internacionales, ahora existe tecnología con la que se pueden analizar variables biométricas de cada atleta (InStat., Wyscout.). En este contexto, surgen algunas interrogantes: a) ¿los datos pasarían a ser de dominio público, y como tales, sujetos al uso indiscriminado de quien los graba?, b) ¿los datos seguirían perteneciendo al deportista?, c) ¿los datos seguirían perteneciendo al club?, o d) ¿los datos provienen de imágenes de la empresa de televisión que adquirió los derechos de transmisión del juego? Ante este escenario, los clubes y las federaciones también 
tienen la responsabilidad de garantizar que los contratos que firman con sus patrocinadores dejen claros los aspectos relacionados con la utilización de los datos personales de cada atleta. Por ejemplo, la empresa Nike. y la Universidad de Michigan en los EE.UU., suscribieron un contrato por \$ 11.6 millones anuales durante 15 años en el que la Universidad de Michigan le otorga a Nike. el derecho de utilizar información biométrica basada en la actividad de cada atleta de variables como la velocidad, distancia, salto vertical, intentos de tiro, posesión del balón, frecuencia cardiaca, ruta de carrera, entre otras. La información a la que se refiere el contrato incluye toda la recolectada por medios digitales de las y los estudiantes-atletas que estuviera relacionada con la fabricación, promoción, mercadeo y ventas de los productos Nike. (Jessop y Baker III, 2019). Por lo tanto, Nike. se apropió de los derechos de los datos. En un caso diferente, la compañía Under Armour. también firmó contratos con al menos siete universidades de EE. UU. para que utilizaran sus dispositivos de recolección de datos biométricos; sin embargo, en los contratos no se especificó a quién pertenecían los datos, o si la empresa podía utilizarlos indiscriminadamente (Jessop y Baker III, 2019).

A pesar de que en EE.UU. existen leyes que protegen los datos de carácter personal, en ese país no existen leyes específicas acerca de la protección de datos biométricos (Pope, 2018; Shahmiri, 2016). Por lo tanto, es necesario reflexionar acerca del tema y proponer legislación nacional que regule este tipo de información personal sensible en el deporte.

\section{Protección de datos en Costa Rica y el caso del Fútbol}

En Costa Rica, existe la ley No 8968, denominada "Protección de la Persona frente al tratamiento de sus datos personales" (Asamblea Legislativa de Costa Rica, 2011). Esta ley tiene su reglamentación e incluso tiene una figura administrativa llamada Agencia de Protección de Datos de los Habitantes (PRODHAB), la cual es responsable, entre otras, de: a) velar por el cumplimiento de la normativa en materia de protección de datos, tanto por parte de personas físicas o jurídicas privadas, como por entes y órganos públicos, b) llevar un registro de las bases de datos reguladas por esta Ley, c) requerir, de quienes administren bases de datos, las informaciones necesarias para el ejercicio de su cargo, entre ellas, los protocolos utilizados, d) acceder a las bases de datos reguladas por esta Ley, a efectos de hacer cumplir efectivamente las normas sobre protección de datos personales, e) resolver sobre los reclamos por infracción a las normas sobre protección de los datos personales, y f) fomentar entre los habitantes el conocimiento de los derechos concernientes al acopio, el almacenamiento, la transferencia y el uso de sus datos personales (http://prodhab.go.cr/funciones/).

La ley No 8968 protege la privacidad de los datos personales de manera muy general, por ejemplo: la dirección del domicilio, el número de teléfono, la dirección de correo electrónico (Rivera, 2019). Sin embargo, en el artículo 9 indica que ninguna persona está obligada a facilitar datos de naturaleza sensible (Asamblea Legislativa de Costa Rica, 2011), entre los cuáles se puede mencionar: el estado de salud, su preferencia sexual, o afiliación religiosa. Los datos biométricos podrían considerarse de naturaleza sensible, debido a que con éstos se podría poner en peligro la adquisición y mantenimiento de un empleo, como es el caso del fútbol profesional (Hernández-Vargas, 2009). Por lo tanto, ¿estarían las y los jugadores de futbol costarricenses en la obligación de permitir la recolección, utilización y almacenamiento de datos biométricos sensibles? Habría primero que definir cuáles datos se considerarían sensibles para las y los jugadores y los clubes, y cuáles no lo serían. Para ello, se debe consultar a profesionales de la Educación Física y el Deporte, así como a otros profesionales. Ejemplos de datos no sensibles podrían ser la estatura o el peso corporal del y la atleta, pero la cantidad de carreras superiores a los $30 \mathrm{~km} / \mathrm{h}$ durante una temporada podrían ser un dato sensible.

Aunque, como se mencionó anteriormente, existe una ley y normativa vigente en la legislación costarricense, sería apropiado incluir la recolección, uso y almacenamiento de datos biométricos para empleos como el de jugador o jugadora profesional de fútbol (u otro deporte), así como cláusulas para el club relacionadas con la ciberseguridad; es decir, con la protección de todos los datos del club ante potenciales 
intrusiones cuyo propósito sea el apropiamiento ilegal de información (hackeo) (Grow y Shackelford, 2020). Cabe mencionar, que en este momento un jugador o jugadora del fútbol profesional costarricense no quedaría en estado de desamparo si se detectara y probara la violación de la propiedad de datos biométricos, pues podría acudir a la PRODHAB e incluso consultar el Título IV, Delitos contra el ámbito de intimidad, del Código Penal de Costa Rica (Gobierno de la República de Costa Rica, 1970) para construir un caso.

Se puede apreciar que, en el ámbito del fútbol, la normativa relacionada con la biometría es peligrosamente escasa, especialmente la relacionada con las obligaciones contractuales entre un club y un jugador o jugadora. El Comité Ejecutivo de la FIFA emitió una serie de lineamientos para la estandarización de los contratos de jugadores de fútbol (Valcke, 2008). La FIFA, como organismo rector del fútbol mundial, indica que, al elaborar un contrato individual, las partes involucradas deben tomar en consideración: "a) la legislación nacional y especialmente las preceptivas cláusulas contractuales..." (Fédération Internationale de Football Association, 2020, p. 2); sin embargo, no se menciona elemento alguno acerca de la propiedad, uso, almacenaje o protección de datos biométricos de naturaleza sensible, lo que podría significar una heterogeneidad de cláusulas en los distintos contratos suscritos entre jugadores y clubes, y entre las diferentes federaciones. Por lo tanto, se deberían emitir regulaciones específicas para normalizar el tema en los contratos.

Por ejemplo, en un contrato real del fútbol profesional costarricense se estipulan múltiples tipos de cláusulas; algunas de ellas relacionadas con la salud y el rendimiento del jugador, así como de mantener absoluta confidencialidad respecto a informaciones técnicas y de otra índole ( Contrato estándar de un jugador de fútbol profesional de Costa Rica, 2020). En ese contrato estándar se menciona que, si el jugador viola la cláusula de confidencialidad, el club considerará como una falta grave al deber de lealtad y fidelidad, con sus respectivas consecuencias ( Contrato estándar de un jugador de fútbol profesional de Costa Rica, 2020). Para ello, el club se acoge a la ley $\mathrm{N}^{\circ} 7975$ Ley de información no divulgada, cuyo objetivo original es proteger la información no divulgada relacionada con los secretos comerciales e industriales (Gobierno de la República de Costa Rica, 2000). Sin embargo, el ámbito de esa ley no incluiría deportistas, pues no menciona las responsabilidades del jugador ni del club con respecto a los datos biométricos de sus atletas.

\section{Conclusiones}

El propósito de este ensayo fue reconocer la importancia de la tecnología portátil en el ámbito del deporte, así como la legislación reguladora asociada en deportistas. Un segundo propósito fue aportar insumos que pueden ser considerados en la creación de planes de estudio dentro de las disciplinas de la Educación Física y Deportes y las Ciencias del Movimiento Humano, entre otras. Se puede concluir que internacionalmente se están haciendo esfuerzos importantes para crear leyes que regulen la recolección y uso de datos sensibles en deportistas. En Costa Rica existe un vacío legal acerca del tema de la recolección, uso, almacenamiento y destrucción de datos biométricos de naturaleza sensible de los deportistas, y específicamente de futbolistas profesionales.

La información sensible recolectada en diversos dispositivos podría convertirse en una poderosa fuente de discordia y de disputas legales si no se incluyen en un contrato, ya que pueden impactar el futuro financiero del jugador y de la organización deportiva que los utiliza. Por ejemplo, un club podría tener a un director técnico que use los datos biométricos para seleccionar jugadores, definir quiénes serían titulares y suplentes, definir la estrategia de juego y realizar ajustes al medio tiempo (Isson, 2018). Por lo tanto, sería recomendable que la FIFA se anticipe y regule esta materia, puesto que se vislumbra como un área de posibles reclamos en un futuro muy cercano, tal y como se observa en la NFL, NBA, MLB, NCAA y otras organizaciones deportivas en los EE.UU. responsables de administrar billones de dólares.

También se vislumbra un crecimiento en la cantidad de publicaciones técnicas y científicas especializadas en el tema discutido en este ensayo desde perspectivas tan diversas como la bioética, la computación e informática, las leyes en el deporte y la bioinformática, entre otras. Finalmente, se espera un crecimiento 
y desarrollo en la oferta académica universitaria en el área de analítica deportiva, para lo cual se deben integrar disciplinas del conocimiento como las ciencias del movimiento humano, medicina, computación e informática, matemática y estadística, derecho, economía, administración e ingeniería, entre otras. Este es un agradable reto para las instituciones de educación superior, constantemente presionadas por la sociedad para ofertar carreras de actualidad y con futuro promisorio. También representa un reto para la formación del personal docente, que debe ser capaz de potenciar sus capacidades a través del estudio constante y la interacción con profesionales de diversas áreas del conocimiento.

\section{AgradeCIMIENTOS}

A la abogada Licda. Margarita Echeverría Bermúdez, por los comentarios realizados a una versión previa de este manuscrito.

\section{ReFERENCIAS}

Arnold, J. y Sade, M. (2017). Wearable technologies in collegiate sports: the ethics of collecting biometric data from student-athletes. The American Journal of Bioethics, 17(1), 67-70. doi: https://doi.org/10.1080/15265161.201 6.1251648

Arnow, G. (2016). Apple watch-ing you: why wearable technology should be federally regulated. Loyola of Los Angeles Law Review, 49, 607-634. Recuperado de https://digitalcommons.lmu.edu/llr/vol49/iss3/2/

Asamblea Legislativa de Costa Rica. (2011). Ley 8968 de Protección de la Persona frente al tratamiento de sus datos personales. San José, Costa Rica: Sistema Costarricense de Información Jurídica. Recuperado de https://bit.ly/ 2Tiwsje

Blanchfield, E., Hargroves, T., Keith, J., Lansing, C., Nordin, H., Palmer, C., ... Napoli, J. (2019). Developing Predictive Athletic Performance Models for Informative Training Regimens. En Industrial Engineering and Operations Management (Presidencia). 2019 Systems and Information Engineering Design Symposium (SIEDS). Conferencia llevada a cabo en el Institute of Integrated Electrical Engineers, Charlottesville, Virginia, USA.

Brush, A. (2015). Investigating the Impact of Using Wearable Technologies during Competitive Sporting Events. Kentucky Alliance for Health, Physical Education, Recreation y Dance Journal, 21-21. Recuperado de https://b it.ly/31CFLiA

Casher, C. (2019). Moneyball in the Era of Biometrics: Who Has Ownership over the Biometric Data of Professional Athletes. Dalhousie Journal of Legal Studies, 28, 1-28. Recuperado de https://bit.ly/3jpqjwq

Chapman, R., Mehta, S., Parent, B. y Caplan, L. (2019). Genetic discrimination: emerging ethical challenges in the context of advancing technology. Journal of Law and the Biosciences, lsz016, 1-23. Recuperado de https://doi. org/10.1093/jlb/lsz016

Contrato estándar de un jugador de fútbol profesional de Costa Rica. (2020). [White paper].

Davalos, S. (2017). Big data has a big role in biostatistics with big challenges and big expectations. Biostatistics and Biometrics Open Access Journal, 1(3), 555-563. doi: https://doi.org/10.19080/BBOAJ.2017.01.555563

Di Tore, A. (2015). Situation awareness and complexity: the role of wearable technologies in sports science. Journal of Human Sport y Exercise, 10, S500-S506. doi: https://doi.org/10.14198/jhse.2015.10.proc1.44

Discover Data Science. (2020). Guide to Choosing a Sports Analytics Degree. Discover Data Science. Recuperado de https://www.discoverdatascience.org/related-programs/sports-analytics/

Emerging Technology from the arXiv. (2016). Big Data Analysis Is Changing the Nature of Sports Science. MIT Technology Review. Recuperado de https://bit.ly/3oiWv8e

Fédération Internationale de Football Association. (2020). Reglamento sobre el estatuto y la transferencia de jugadores. Zúrich, Suiza: Fédération Internationale de Football Association (FIFA). 
Gil, E. (2016). Big data, privacidad y protección de datos. Madrid: Ministerio de la Presidencia: Agencia Estatal Boletín Oficial del Estado. Recuperado de https://www.aepd.es/sites/default/files/2019-10/big-data.pdf

Gobierno de la República de Costa Rica. (1970). Código Penal de Costa Rica. Título IV, Delitos contra el ámbito de intimidad, Ley $N^{\circ}$ 4573. San José, Costa Rica: Imprenta Nacional. Recuperado de https://bit.ly/2Ti4Jzg

Gobierno de la República de Costa Rica. (2000). Ley de Información No Divulgada N 7975. San José, Costa Rica: Sistema Costarricense de Información Jurídica. Recuperado de https://bit.ly/3kriNCv

Greenbaum, D. (2018). Wuz You Robbed? Concerns With Using Big Data Analytics in Sports. American Journal of Bioethics, 18(6), 32-33. doi: https://doi.org/10.1080/15265161.2018.1459953

Greenwald, M. (2017). Cybersecurity in Sports. Questions of Privacy and Ethics. Tufts University Department of Computer Science. Recuperado de http://www.cs.tufts.edu/comp/116/archive/fall2017/mgreenwald.pdf

Grow, N. y Shackelford, S. (2020). The Sport of Cybersecurity: How Professional Sports Leagues Can Better Protect the Competitive Integrity of Their Games. Boston College Law Review, 61, 1-56. Recuperado de https://lawdi gitalcommons.bc.edu/bclr/vol61/iss $2 / 3 /$

Hand, D. (2018). Aspects of Data Ethics in a Changing World: Where Are We Now? Big Data, 6(3), 176-190. doi: https://doi.org/10.1089/big.2018.0083

Harriss, J., MacSween, A. y Atkinson, G. (2019). Ethical Standards in Sport and Exercise Science Research: 2020 Update. Int J Sports Med, 40(13), 813-817. doi: https://doi.org/10.1055/a-1015-3123

Hattery, M. (2017). Major League Baseball players, big data, and the right to know: the duty of major league baseball teams to disclose health modeling analysis to their players. Marquette Sports Law Review, 28(1), 257-283. Recuperado de https://bit.ly/3mnZuKZ

Henne, K. (2017). "I Felt Like a Lab Rat": The Importance of Power and Context in Understanding Biometric Technologies. The American Journal of Bioethics, 17(1), 63-65. doi: https://doi.org/10.1080/15265161.2016. 1251659

Hernández-Vargas, A. M. (2009). Ordenamiento Jurídico Laboral-Deportivo de los Jugadores Profesionales de Fútbol en Costa Rica. (Tesis de Licenciatura). Universidad de Costa Rica, San José, Costa Rica.

Inglés, P., Pino, J., Bastida, A. y Gómez C. D. (2018). Análisis cinemático de las exigencias en futbolistas de categoría benjamín mediante un dispositivo inercial (WIMU $\mathrm{PRO}^{\mathrm{TM}}$ ). Kronos: revista universitaria de la actividad fisica y el deporte, 17(1), 1-10. Recuperado de https://bit.ly/3dS4MLz

Isson, P. (2018). Unstructured Data Analytics: How to Improve Customer Acquisition, Customer Retention, and Fraud Detection and Prevention. Hoboken, NJ, EE.UU.: John Wiley y Sons, Inc.

Jessop, A. y Baker III, A. (2019). Big Data Bust: Evaluating the Risks of Tracking NCAA Athletes' Biometric Data. Texas Review of Entertainment y Sports Law, 20(1), 81-112. Recuperado de https://bit.ly/3mi8QaZ

Jhajharia, S., Pal, S. y Verma, S. (2014). Wearable computing and its application. International Journal of Computer Science and Information Technologies, 5(4), 5700-5704. Recuperado de https://bit.ly/3kr0Sf8

Kampakis, S. (2016). Predictive modelling of football injuries. (Tesis de doctorado). University College London. London, UK. Recuperado de https://arxiv.org/ftp/arxiv/papers/1609/1609.07480.pdf

Kannan, A., Kolovich, B., Lawrence, B. y Rafiqi, S. (2018). Predicting National Basketball Association Success: A Machine Learning Approach. SMU Data Science Review, 1(3), 1-15. Recuperado de https://scholar.smu.edu/d atasciencereview/vol1/iss3/7/

Karkazis, K. y Fishman, J. R. (2017). Tracking US professional athletes: The ethics of biometric technologies. The American Journal of Bioethics, 17(1), 45-60. doi: https://doi.org/10.1080/15265161.2016.1251633

Lamkin, P. (2016). Wearable Tech Market To Be Worth \$34 Billion By 2020. Forbes. Recuperado de https://bit.ly /31ACYX4

Lazan, A. y Greenbaum, D. (2017). Collegiate sports: professionals all but in name raise unique bioethics concerns in the collection of biometric data. The American Journal of Bioethics, 17(1), 70-72. doi: https://doi.org/10.1080 $/ 15265161.2016 .1251646$ 
Lemire, J. (2016). Baseball's Union Remains Wary of Wearables. Players are concerned that their personal health data is the next moneyball frontier. Vocativ. Recuperado de https://bit.ly/3jlH0sl

Major League of Soccer. (2019). 2019 MLS Rosters. Recuperado de https://www.mlssoccer.com/rosters/2019

Morgulev, E., Azar, H. y Lidor, R. (2018). Sports analytics and the big-data era. International Journal of Data Science and Analytics, 5(4), 213-222. doi: https://doi.org/10.1007/s41060-017-0093-7

Osborne, B. (2017). Legal and Ethical Implications of Athletes' Biometric Data Collection in Professional Sport. Marquette Sports Law Review, 28(1), 37-84. Recuperado de https://bit.ly/3jrhjqz

Pope, C. (2018). Biometric data collection in an unprotected world: Exploring the need for federal legislation protecting biometric data. Journal of Law and Policy, 26(2), 769-803. Recuperado de https://brooklynworks.b rooklaw.edu/cgi/viewcontent.cgi?article $=1570$ ycontext $=$ jlp

Rein, R. y Memmert, D. (2016). Big data and tactical analysis in elite soccer: future challenges and opportunities for sports science. SpringerPlus, 5(1), 1410. doi: https://doi.org/10.1186/s40064-016-3108-2

Rivera, V. (2019). Realidad sobre la Privacidad de los Datos Personales en Costa Rica. e-Ciencias de la Información, 9(2), 3-12. doi: https://doi.org/10.15517/ECI.V9I2.37503

Roberts, L., Cohen, I. G., Deubert, R. y Lynch, F. (2016). Evaluating NFL player health and performance: legal and ethical issues. University of Pennsylvania Law Review, 165(2), 227-314. Recuperado de https://scholarship.law .upenn.edu/penn_law_review/vol165/iss2/1/

Rossi, A., Pappalardo, L., Cintia, P., Iaia, M., Fernàndez, J. y Medina, D. (2018). Effective injury forecasting in soccer with GPS training data and machine learning. PLOS ONE, 13(7), 1-15. doi: https://doi.org/10.1371/journal .pone.0201264

Rotnitzky, N. (2019). Entrevista a Adrián Vaccarini (Preparador físico FPF): El método de Gareca para llevar a Perú a un Mundial y a la final de la Copa América. Analitica Sports. Recuperado de https://bit.ly/35wDbLY

Saxon, A. (2017). Athletic Performance Monitoring, Pseudo Science and Metaphysics Meet Ethics. The American Journal of Bioethics, 17(1), 61-62. doi: https://doi.org/10.1080/15265161.2016.1251663

Schulz, M. y Hennis J. A. (2016). Regulation (EU) 2016/679 of the European Parliament and of the Council. Brussels: Official Journal of the European Union

Shahmiri, S. (2016). Wearing Your Data on Your Sleeve: Wearables, the FTC, and the Privacy Implications of this New Technology. Texas Review of Entertainment y Sports Law, 18(1), 25-48. Recuperado de https://bit.ly/2H qnvBL

Sikka, S., Baer, M., Raja, A., Stuart, M. y Tompkins, M. (2019). Analytics in sports medicine: implications and responsibilities that accompany the era of big data. The Journal of Bone y Joint Surgery, 101(3), 276-283. doi: h ttps://doi.org/10.2106/JBJS.17.01601

Socolow, R. y Jolly, I. (2017). Game-changing wearable devices that collect athlete data raise data ownership issues. World Sports Advocate, 15(7), 15-17. Recuperado de https://bit.ly/2Hw3IB2

Valcke, J. (2008). Circular $N^{\circ} 1171$. Requisitos mínimos para contratos estándar de jugadores de fútbol profesional. En Federación Internacional de Fútbol Asociación (Ed.). Zúrich, Suiza: Federación Internacional de Fútbol Asociación (FIFA).

Vermeulen, E. y Venkata, S. (2018). Big data in sport analytics: applications and risks. En Industrial Engineering and Operations Management (Presidencia). Proceedings of the International Conference on Industrial Engineering and Operations Management. Conferencia llevada a cabo en IEOM Society International. Pretoria/Johannesburg, South Africa. Recuperado de https://bit.ly/3ojotk9 